\title{
The number of independent sets in a connected graph and its complement
}

\author{
Yumei $\mathrm{Hu}^{*}$, Yarong Wei \\ School of Mathematics, Tianjin University, Tianjin, PR China
}

Received 29 March 2017, accepted 3 January 2018, published online 26 July 2018

\begin{abstract}
For a connected graph $G$, the total number of independent vertex sets (including the empty vertex set) is denoted by $i(G)$. In this paper, we consider Nordhaus-Gaddum-type inequalities for the number of independent sets of a connected graph with connected complement. First we define a transformation on a graph that increases $i(G)$ and $i(\bar{G})$. Next, we obtain the minimum and maximum value of $i(G)+i(\bar{G})$, where graph $G$ is a tree $T$ with connected complement and a unicyclic graph $G$ with connected complement, respectively. In each case, we characterize the extremal graphs. Finally, we establish an upper bound on the $i(G)$ in terms of the Wiener polarity index.

Keywords: Independent sets, connected complement, bounds, the Wiener polarity index, NordhausGaddum-type inequality.
\end{abstract}

Math. Subj. Class.: 05C69, 05C30

\section{Introduction}

Let $G=(V(G), E(G))$ be a simple connected graph of order $n$ with vertex set $V(G)$ and edge set $E(G)$, denote by $N_{G}(u)$ the set of neighbors of a vertex $u$ in $G$, and denote by $G[S]$ the graph which is induced by vertex set $S \subseteq V(G)$. A double star $S_{p, q}$ is obtained from $S_{p}$ and $S_{q}$ by connecting the center of $S_{p}$ with that of $S_{q}$. A graph is unicyclic if and only if it is connected and has size equal to its order.

Given a graph $G$, a $k$-independent set is a set of $k$ vertices, no two of which are adjacent. Denote by $i(G ; k)$ the number of $k$-independent sets of $G, k \geq 1$. It is both consistent and convenient to define $i(G ; 0)=1$. The family of the independent sets in $G$ which contains the vertex sets $U$ and $S$ is denoted by $I_{U, S}(G)$, and let $i_{U, S}(G ; k)$ be its cardinality. The

* The corresponding author, Yumei Hu, supported by NSFC NO. 11001196.

E-mail addresses: huyumei@tju.edu.cn (Yumei Hu), math5025@163.com (Yarong Wei) 
total number of independent vertex sets (including the empty vertex set) of a molecular graph $G=(V, E)$, denoted by $i(G)$, is defined as

$$
i(G)=\sum_{k \geq 0} i(G ; k) .
$$

In chemical literature, the number of the independent sets of graphs $i(G)$ is referred to as the Merrifield-Simmons index. It is a valuable topological index introduced by the American chemists Richard E. Merrifield and Howard E. Simmons [12] in 1989. It is one of the topological indices whose mathematical characteristics has been extensively studied in a monograph $[11,20]$. Its applicability for QSPR and QSAR was also examined to a much lesser extent. In [12] it has been shown that $i(G)$ is correlated with boiling points. And, for the path $P_{n}, i\left(P_{n}\right)$ is equal to the Fibonacci number $F_{n+1}$ [15].

The problem of counting the number of independent sets in a graph is NP-complete [16]. However, for certain types of graphs the problem of determining their number of independent subsets is polynomial. For instance, the number of independent sets in tree, uncyclic, and tricyclic graphs are calculated in [15, 14, 21], respectively. It is of significant interest to study the extremal graphs having maximal or minimal index. Zhu [20] characterized the extremal unicyclic graphs with a perfect matching which have maximal, second maximal Merrifield-Simmons index. In [17], S. Wagner and I. Gutman wrote a survey of results and techniques on the Hosoya index and Merrifield-Simmons index. Other recent results on the number of independent sets can be found in [2, 4, 3, 9].

The number of unordered vertices pairs that are at distance 3 in a graph $G$, denoted by $W_{p}(G)$, is

$$
W_{p}(G)=\left|\left\{(u, v) \mid d_{G}(u, v)=3, u, v \in V(G)\right\}\right| .
$$

It is also referred as the Wiener polarity index ([5, 7, 8]). Motivated by the result of [7], Hua et al. gave an upper bound on the Wiener polarity index in terms of the Hosoya index. We can find that, in a graph $G$, every pair of vertices at distance 3 corresponds to some 2 -independent sets. There are also some relationships between the number of independent sets and the Wiener polarity index.

The Nordhaus-Gaddum-type results are bounds of the sum or the product of a parameter for a graph and its complement. The name "Nordhaus-Gaddum-type" is given because Nordhaus and Gaddum [13] first found this type of inequality for the chromatic number of a graph and its complement in 1956. Since then, Nordhaus-Gaddum-type inequalities for many other graph invariants have been studied in a number of papers [1, 7, 10, 19]. We respectively research Nordhaus-Gaddum-type results for tree $i(T)$, unicyclic graph $i(G)$ and connected graph $i(G)$.

In this paper, we consider Nordhaus-Gaddum-type inequalities for the number of independent sets of a connected graph with a connected complement. Firstly, in Section 2 we establish a transformation on graphs that increases $i(G)$ and $i(\bar{G})$. Secondly, in Section 3 and 4 , we obtain the minimum and maximum value of $i(G)+i(\bar{G})$, where graph $G$ is a tree $T$ with connected complement and a unicyclic graph $G$ with connected complement, respectively. In each case, we characterize the extremal graphs. Finally, in Section 5 we establish a lower bound on $i(G)$ in terms of the Wiener polarity index. And, for a connected graph $G$ with connected complement $\bar{G}$, we obtain the minimum of $i(G)+i(\bar{G})$. Also, we pose a conjecture about which graph obtains the maximum value of $i(G)+i(\bar{G})$.

Other notation and terminology not defined here will conform to those in [18]. 


\section{Preliminary}

Lemma 2.1 ([6]). Let $G=(V, E)$ be a graph.

(1) If $u v \in E(G)$, then $i(G)=i(G-u v)-i(G-(N[u] \cup N[v]))$;

(2) If $u \in V(G)$, then $i(G)=i(G-u)+i(G-N[u])$;

(3) If $G_{1}, G_{2}, \ldots, G_{t}$ are the components of the graph $G$, then $i(G)=\prod_{j=1}^{t} i\left(G_{j}\right)$.

Theorem 2.2. Let $G$ be a simple graph and uv an edge of $G$ such that $N_{G}(u) \cap N_{G}(v)=\emptyset$ and $d(u), d(v)>1$. Let $G_{u, v}$ denote the graph obtained from $G$ by identifying vertex $u$ and $v$ (the new vertex is labeled as $u$ ) and attaching a pendent vertex $v$ at $u$. Then

(1) $i\left(\overline{G_{u, v}}\right) \geq i(\bar{G})$ with equality if and only if $\bar{G}\left[N_{G-u}(v) \cup N_{G}(u) \backslash\{v\}\right]$ is not an empty graph;

(2) $i\left(G_{u, v}\right) \geq i(G)$ with equality if and only if $G\left[N_{G-u}(v) \cup N_{G}(u) \backslash\{v\}\right]$ is not an empty graph.

Proof. For convenience, let $G^{\prime}=G_{u, v}$. By Lemma 2.1(1), for all non-negative integers $k$, we have $i(G ; k)=i(G-u ; k)+i\left(G-u-N_{G}(u) ; k-1\right)$.

(1) By $i(G ; k)=i(G-u ; k)+i\left(G-u-N_{G}(u) ; k-1\right)$, we have

$$
\begin{aligned}
& i(\bar{G} ; k)=i(\bar{G}-v ; k)+i\left(\bar{G}-v-N_{\bar{G}}(v) ; k-1\right) \\
& \quad=i(\bar{G}-v-u ; k)+i\left(\bar{G}-v-u-N_{\bar{G}-v}(u) ; k-1\right)+i\left(\bar{G}-v-N_{\bar{G}}(v) ; k-1\right)
\end{aligned}
$$

and

$$
\begin{aligned}
i\left(\overline{G^{\prime}} ; k\right)=i\left(\overline{G^{\prime}}-v-u ; k\right)+i\left(\overline{G^{\prime}}-v-u-N_{\overline{G^{\prime}}-v}(u) ; k-1\right) & \\
& +i\left(\overline{G^{\prime}}-v-N_{\overline{G^{\prime}}}(v) ; k-1\right) .
\end{aligned}
$$

Obviously,

$$
\begin{aligned}
\bar{G}-v-u & =\overline{G^{\prime}}-v-u, \\
N_{\bar{G}-v}(u) & =N_{\overline{G^{\prime}}-v}(u) \cup\left(N_{G}(v) \backslash\{u\}\right), \\
N_{\overline{G^{\prime}}}(v) & =N_{\bar{G}}(v) \cup\left(N_{G}(v) \backslash\{u\}\right), \\
N_{\bar{G}}(v) & =\left[N_{\bar{G}}(u) \backslash\left(N_{G}(v) \backslash\{u\}\right)\right] \cup\left[N_{G}(u) \backslash\{v\}\right], \\
\overline{G^{\prime}}-v-N_{\overline{G^{\prime}}}(v) & =\bar{G}-v-N_{\bar{G}}(v)-\left(N_{G}(v) \backslash\{u\}\right), \\
N_{\bar{G}}(v) \cap\left(N_{G}(v) \backslash\{u\}\right) & =\emptyset, \text { and } \\
N_{\overline{G^{\prime}}-v}(u) \cap\left(N_{G}(v) \backslash\{u\}\right) & =\emptyset .
\end{aligned}
$$

So,

$$
\begin{aligned}
i\left(\overline{G^{\prime}} ; k\right)- & i(\bar{G} ; k) \\
= & i(\bar{G}-v-u ; k)+i\left(\bar{G}-v-N_{\bar{G}}(v)-\left(N_{G}(v) \backslash\{u\}\right) ; k-1\right) \\
+ & i\left(\bar{G}-v-u-\left[N_{\bar{G}}(u) \backslash\left(N_{G}(v) \backslash\{u\}\right)\right] ; k-1\right)-i(\bar{G}-v-u ; k) \\
& -i\left(\bar{G}-v-u-N_{\bar{G}-v}(u) ; k-1\right)-i\left(\bar{G}-v-N_{\bar{G}}(v) ; k-1\right) \\
= & i_{N_{G}(v) \backslash\{u\}}\left(\bar{G}-v-u-\left[N_{\bar{G}-v}(u) \backslash\left(N_{G}(v) \backslash\{u\}\right)\right] ; k-1\right) \\
- & -i_{N_{G}(v) \backslash\{u\}}\left(\bar{G}-v-N_{\bar{G}}(v) ; k-1\right)
\end{aligned}
$$




$$
\begin{gathered}
=i_{N_{G}(v) \backslash\{u\}}\left(\bar{G}-v-u-\left[N_{\bar{G}-v}(u) \backslash\left(N_{G}(v) \backslash\{u\}\right)\right] ; k-1\right) \\
-i_{N_{G}(v) \backslash\{u\}}\left(\bar{G}-v-u-N_{\bar{G}}(v) ; k-1\right) \\
-i_{N_{G}(v) \backslash\{u\}}\left(\bar{G}-v-u-N_{\bar{G}}(v)-N_{\bar{G}-v-N_{\bar{G}}(v)}(u) ; k-2\right) \\
=i_{N_{G}(v) \backslash\{u\}, N_{G}(u) \backslash\{v\}}\left(\bar{G}-v-u-\left[N_{\bar{G}-v}(u) \backslash\left(N_{G}(v) \backslash\{u\}\right)\right] ; k-1\right) \geq 0 .
\end{gathered}
$$

Obviously, if

$$
i_{N_{G}(v) \backslash\{u\}, N_{G}(u) \backslash\{v\}}\left(\bar{G}-v-u-\left[N_{\bar{G}-v}(u) \backslash\left(N_{G}(v) \backslash\{u\}\right)\right] ; k-1\right)=0,
$$

$\bar{G}\left[N_{G-u}(v) \cup N_{G}(u) \backslash\{v\}\right]$ is not an empty graph. Conversely, if graph $\bar{G}\left[N_{G-u}(v) \cup\right.$ $\left.N_{G}(u) \backslash\{v\}\right]$ is not an empty graph,

$$
i_{N_{G}(v) \backslash\{u\}, N_{G}(u) \backslash\{v\}}\left(\bar{G}-v-u-\left[N_{\bar{G}-v}(u) \backslash\left(N_{G}(v) \backslash\{u\}\right)\right] ; k-1\right)=0 .
$$

(2) By $i(G ; k)=i(G-u ; k)+i\left(G-u-N_{G}(u) ; k-1\right)$, we can similarly get:

$$
\begin{aligned}
& i(G ; k)=i(G-u ; k)+i\left(G-u-N_{G}(u) ; k-1\right) \\
& \quad=i(G-u-v ; k)+i\left(G-u-v-N_{G-u}(v) ; k-1\right)+i\left(G-u-N_{G}(u) ; k-1\right)
\end{aligned}
$$

and

$$
\begin{array}{r}
i\left(G^{\prime} ; k\right)=i\left(G^{\prime}-u-v ; k\right)+i\left(G^{\prime}-u-v-N_{G^{\prime}-u}(v) ; k-1\right) \\
+i\left(G^{\prime}-u-N_{G^{\prime}}(u) ; k-1\right) .
\end{array}
$$

Obviously,

$$
\begin{aligned}
G-u-v & =G^{\prime}-u-v, \\
N_{G^{\prime}-u}(v) & =\emptyset, \\
N_{G}(u) \cap N_{G-u}(v) & =\emptyset, \text { and } \\
G^{\prime}-u-N_{G^{\prime}}(u) & =G-u-N_{G}(u)-N_{G-u}(v) .
\end{aligned}
$$

So,

$$
\begin{gathered}
i\left(G^{\prime} ; k\right)-i(G ; k)=i(G-u-v ; k)+i\left(G-u-N_{G}(u)-N_{G-u}(v) ; k-1\right) \\
+i(G-u-v ; k-1)-i\left(G-u-v-N_{G-u}(v) ; k-1\right) \\
-i(G-u-v ; k)-i\left(G-u-N_{G}(u) ; k-1\right) \\
=i_{N_{G-u}(v)}(G-u-v ; k-1)-i_{N_{G-u}(v)}\left(G-u-N_{G}(u) ; k-1\right) \\
=i_{N_{G-u}(v), N_{G}(u) \backslash\{v\}}(G-u-v ; k-1) \geq 0 .
\end{gathered}
$$

Obviously, if

$$
i_{N_{G-u}(v), N_{G}(u) \backslash\{v\}}(G-u-v ; k-1)=0,
$$

$G\left[N_{G-u}(v) \cup N_{G}(u) \backslash\{v\}\right]$ is not an empty graph. Conversely, if graph $G\left[N_{G-u}(v) \cup\right.$ $\left.N_{G}(u) \backslash\{v\}\right]$ is not an empty graph,

$$
i_{N_{G-u}(v), N_{G}(u) \backslash\{v\}}(G-u-v ; k-1)=0 .
$$




\section{The Nordhaus-Gaddum-type inequality for trees}

In this section, we consider a tree $T$ with connected complement $\bar{T}$, then we obtain the minimum and maximum value of $i(T)+i(\bar{T})$ and characterize the extremal graph.

Lemma 3.1 ([15]). The star $S_{n}$ has the maximal Merrifield-Simmons index for all trees with $n$ vertices. And, the path $P_{n}$ has the minimal Merrifield-Simmons index for all trees with $n$ vertices.

For the proof, we give an equality involving $i(T)+i(\bar{T})$ as follows.

Lemma 3.2. Let $T$ be a tree of order $n$ with connected complement $\bar{T}$. Then

$$
i(T)+i(\bar{T})=2 n+i(T) .
$$

Proof. For connected complement $\bar{T}$ and all non-negative integers $k \geq 3$, it is easy to verify $i(\bar{T} ; k)=0$ and $i(\bar{T} ; 2)=|E(T)|=n-1$. Therefore

$$
i(T)+i(\bar{T})=i(T)+1+n+i(\bar{T} ; 2)=2 n+i(T) .
$$

Now we give the Nordhaus-Gaddum-type inequality of a tree for $i(T)$.

Theorem 3.3. Let $T$ be a tree of order $n$ with connected complement $\bar{T}$, then

$$
i(T)+i(\bar{T}) \geq 2 n+F_{n+1}
$$

with equality if and only if $T \cong P_{n}$, where $F_{n+1}$ is the Fibonacci number.

Proof. By Lemma 3.1 and Lemma 3.2 graph which reaches the minimum value of $i(T)+$ $i(\bar{T})$.

And, $i\left(P_{n}\right)$ is equal to the Fibonacci number $F_{n+1}$, then $i(T)+i(\bar{T}) \geq 2 n+F_{n+1}$.

Theorem 3.4. Let $T$ be a tree of order $n$ with connected complement $\bar{T}$, then

$$
i(T)+i(\bar{T}) \leq 2+2 n+2^{n-3}+2^{n-2}
$$

with equality if and only if $T \cong S_{2, n-2}$.

Proof. If $T$ and $\bar{T}$ are connected graphs, then the star $S_{n}$ is not the extremal graph which reaches the maximum value of $i(T)+i(\bar{T})$. So we assume $D(T) \geq 3$.

Let $P=v_{0} v_{1} \ldots v_{D(T)}$ be a diametrical path of tree $T$. By Theorem 2.2, we have

$$
i\left(T_{D(T)-1, D(T)-2}\right)+i\left(\overline{T_{D(T)-1, D(T)-2}}\right)>i(T)+i(\bar{T}) .
$$

Obviously, graph $T_{D(T)-1, D(T)-2}$ is a tree of order $n$.

Therefore, for the tree of order $n$ with connected complement, by shortening the diametrical path of a tree, we can get the extremal graph the double star $S_{p, q}$ which reaches the maximal value of $i(T)+i(\bar{T})$. For the double star $S_{p, q}$ of order $n$, we have

$$
\begin{aligned}
i\left(S_{p, q}\right)+i\left(\overline{S_{p, q}}\right) & =2+2 n+\sum_{i=2}^{n-2}\left(\begin{array}{c}
n-2 \\
i
\end{array}\right)+\sum_{i=1}^{p-1}\left(\begin{array}{c}
p-1 \\
i
\end{array}\right)+\sum_{i=1}^{q-1}\left(\begin{array}{c}
q-1 \\
i
\end{array}\right)+p+q-1 \\
& =2 n+2^{n-2}+2^{p-1}+2^{q-1} \\
& \leq 2+2 n+2^{n-3}+2^{n-2}
\end{aligned}
$$

with equality if and only if $p=n-2$ or $q=n-2$. So $i(T)+i(\bar{T}) \leq 2+2 n+2^{n-3}+2^{n-2}$ with equality if and only if $T \cong S_{2, n-2}$. 


\section{The Nordhaus-Gaddum-type inequality for unicyclic graphs}

In this section, we consider a unicyclic graph $G$ of order $n$ with connected complement $\bar{G}$, then we obtain the minimum and maximum value of $i(G)+i(\bar{G})$ and characterize the extremal graph. Obviously, if $n<5$, any complement $\bar{G}$ is not connected. We need to consider the case when $n \geq 5$.

Lemma 4.1 ([14]). If $G$ is a unicyclic graph of order $n$, then

(1) $i(G) \geq F_{n-1}+F_{n+1}$ and equality occurs if and only if $G \cong C_{n}$ or $G \cong L_{n, 3}$, where $L_{n, 3}$ is the unicyclic graph of order $n$ obtained from the two vertex disjoint graphs $C_{3}$ and $P_{n-3}$ by adding an edge joining a vertex of $C_{3}$ to an endvertex of $P_{n-3}$.

(2) $i(G) \leq 3 \times 2^{n-3}+1$ and equality holds if and only if $G$ is a 4-cycle or $G \cong H_{n, 3}$, where $H_{n, 3}$ is the unicyclic graph of order $n$ constructed by attaching $n-3$ leaves to one vertex on a cycle of length 3.

For the proof, we give an equality about $i(G)+i(\bar{G})$ as follows.

Lemma 4.2. Let $G$ be a unicyclic graph of order $n \geq 5$ with connected complement $\bar{G}$, then

$$
i(G)+i(\bar{G})=1+2 n+i(\bar{G} ; 3)+i(G) .
$$

Proof. For connected complement $\bar{G}$ and all non-negative integers $k \geq 4$, it is easy to verify $i(\bar{G} ; k)=0$ and $i(\bar{G} ; 2)=|E(G)|=n$. Therefore

$$
i(G)+i(\bar{G})=i(G)+1+n+i(\bar{G} ; 2)+i(\bar{G} ; 3)=1+2 n+i(\bar{G} ; 3)+i(G) .
$$

Now we give the Nordhaus-Gaddum-type inequality of a unicyclic graph for $i(G)$.

Theorem 4.3. Let $G$ be a unicyclic graph of order $n \geq 5$ with connected complement $\bar{G}$, then

$$
i(G)+i(\bar{G}) \geq 1+2 n+F_{n-1}+F_{n+1}
$$

with equality if and only if $G \cong C_{n}$, where $F_{n+1}$ is the Fibonacci number.

Proof. Obviously, $i\left(\overline{L_{n, 3}} ; 3\right)=1>i\left(\overline{C_{n}} ; 3\right)=0$, and the complement of graph $C_{n}$ is a connected graph. Then by Lemma 4.1(1) and Lemma 4.2, we have $i\left(L_{n, 3}\right)=i\left(C_{n}\right)$ and

$$
\begin{aligned}
i(G)+i(\bar{G}) & =1+2 n+i(\bar{G} ; 3)+i(G) \\
& \geq 1+2 n+i\left(\overline{C_{n}} ; 3\right)+i\left(C_{n}\right) \\
& =1+2 n+F_{n-1}+F_{n+1} .
\end{aligned}
$$

In order to formulate our results, some graphs need to be defined. Let $O_{x_{1}, x_{2}, x_{3}}$ denote a unicyclic graph on $n$ vertices created from a cycle $C_{3}=v_{1} v_{2} v_{3}$ by attaching $x_{i}(i=1,2,3)$ pendent vertices to $v_{i}$ such that $x_{1}+x_{2}+x_{3}+3=n$ and $x_{1} \geq x_{2} \geq x_{3}, x_{2} \geq 1$.

Let $U_{y_{1}, y_{2}}$ denote a unicyclic graph on $n$ vertices created from a cycle $C_{3}=v_{1} v_{2} v_{3}$ by attaching $y_{1}$ pendent vertices $u_{1}, u_{2}, \ldots, u_{y_{1}}$ to $v_{1}$ and attaching $y_{2}$ pendent vertices to $u_{1}$ such that $y_{1}+y_{2}+3=n$ and $y_{1} \geq 1, y_{2} \geq 2$.

Theorem 4.4. Let $G$ be a unicyclic graph of order $n \geq 5$ with connected complement $\bar{G}$, then

$$
i(G)+i(\bar{G}) \leq 4+2 n+2^{n-4}+2^{n-2}
$$

with equality if and only if $G \cong O_{n-4,1,0}$. 
Proof. If the cycle in $G$ is of length greater than three, then by applying the transformation in Theorem 2.2 to the cycle, there is a unicyclic graph $L_{1}$ with a triangle such that $i\left(L_{1}\right)+$ $i\left(\overline{L_{1}}\right)>i(G)+i(\bar{G})$.

Let $\mathcal{H}$ denote the set of all unicyclic graphs $H$ with a triangle. Then for all $H \in \mathcal{H}$ by Lemma 4.2 we have $i(H)+i(\bar{H})=1+2 n+i(\bar{H} ; 3)+i(H)=2+2 n+i(H)$. The maximum value of $i(H)+i(\bar{H})$ is equal to the maximum value of $i(H)$. By Lemma 4.1(2), we know that the graph $H_{n, 3}$ is the extremal graph which obtains the maximum value $i(H)$, but the graph $\overline{H_{n, 3}}$ is not connected. So we calculate the second maximum value of $i(H)$.

Case 1: There is a vertex in $H$ with distance at least two to the 3-cycle.

By the transformation in Theorem 2.2, we get there is a graph $L_{2}$ with $i\left(L_{2}\right)>i(H)$, where $L_{2} \cong O_{x_{1}, x_{2}, x_{3}}$ or $L_{2} \cong U_{y_{1}, y_{2}}$.

$$
\begin{aligned}
i\left(U_{y_{1}, y_{2}}\right) & =i\left(U_{y_{1}, y_{2}}-v_{3}\right)+i\left(U_{y_{1}, y_{2}}-N_{U_{y_{1}, y_{2}}}\left[v_{3}\right]\right) \\
& =i\left(S_{y_{1}+1, y_{2}+1}\right)+i\left(K_{y_{1}-1} \cup S_{y_{2}+1}\right) \\
& =3 \times 2^{n-4}+2^{y_{2}}+3 \times 2^{y_{1}-1} \\
& \leq 3 \times 2^{n-4}+2+3 \times 2^{n-5} \\
& <2+2^{n-4}+2^{n-2}=i\left(O_{n-4,1,0}\right)
\end{aligned}
$$

Case 2: $G \cong O_{x_{1}, x_{2}, x_{3}}$

$$
\begin{aligned}
i\left(O_{x_{1}, x_{2}, x_{3}}\right) & =i\left(O_{x_{1}, x_{2}, x_{3}}-v_{3}\right)+i\left(O_{x_{1}, x_{2}, x_{3}}-N_{O_{x_{1}, x_{2}, x_{3}}}\left[v_{v_{3}}\right]\right) \\
& =i\left(S_{x_{1}+1, x_{2}+2} \cup \overline{K_{x_{3}}}\right)+i\left(\overline{K_{x_{1}+x_{2}}}\right) \\
& =2^{n-3}+2^{x_{1}+x_{3}}+2^{x_{2}+x_{3}}+2^{n-3-x_{3}} \\
& \leq 2^{n-3}+2^{x_{1}+x_{3}}+2^{x_{2}}+2^{n-3}=i\left(O_{x_{1}+x_{3}, x_{2}, 0}\right) \\
& \leq 2^{n-3}+2^{n-4}+2+2^{n-3}=i\left(O_{n-4,1,0}\right)
\end{aligned}
$$

with equality if and only if $x_{3}=0$ and $x_{2}=1$. Obviously, the graph $\overline{O_{n-4,1,0}}$ is connected. So $i(G)+i(\bar{G}) \leq i(H)+i(\bar{H}) \leq 4+2 n+2^{n-4}+2^{n-2}$ with equality if and only if $G \cong O_{n-4,1,0}$.

\section{The Nordhaus-Gaddum-type inequality for connected graphs}

In this section, we obtain a lower bound on $i(G)$ in terms of the Wiener polarity index. And, for a connected graph $G$ with connected complement $\bar{G}$, we obtain a minimum value of $i(G)+i(\bar{G})$ and characterize the extremal graph. Also, we pose a conjecture about which graph gets the maximum value of $i(G)+i(\bar{G})$.

Lemma 5.1 ([7]). Let $G$ be a connected graph with connected complement $\bar{G}$, then

$$
W_{p}(G)+W_{p}(\bar{G}) \geq D(G)+D(\bar{G})-4 \text {. }
$$

Moreover, equality holds if and only if $G \cong P_{n}$ or $G \cong G^{* *}$ or $D(G)=D(\bar{G})=2$. The graph $G^{* *}$ of order $n \geq 5$ is obtained from a path $P_{4}$ by joining each vertex of $H_{n-4}$ to each internal vertex of the path $P_{4}$ such that $V\left(G^{* *}\right) \backslash V\left(P_{4}\right)=V\left(H_{n-4}\right)$, where $H_{n-4}$ is any graph of order $n-4$. 
In order to get the lower bound on the $i(G)+i(\bar{G})$, we give a lower bound on the $i(G)$ in terms of the Wiener polarity index.

Lemma 5.2. Let $G$ be a connected graph of order $n$ and $D(G) \geq 2$. Then

$$
i(G) \geq 2+n+2 W_{p}(G)
$$

with equality if and only if $G \cong G_{n}$ or $G \cong B_{2, n-2}$, where $G_{n}=K_{n}-e, e \in E\left(K_{n}\right)$, $B_{2, n-2}$ is a graph on $n \geq 3$ vertices obtained from $P_{2}$ and $K_{n-2}$ by coinciding any vertex of $P_{2}$ with that of $K_{n-2}$.

Proof. If $D(G)=2$, then $W_{p}(G)=0$. Let $P=u x y$ be a diametrical path, then $\{u, y\}$ is a 2 -independent set of $G$. Therefore

$$
i(G) \geq i(G ; 0)+i(G ; 1)+i(G ; 2) \geq 1+n+1=2+n+2 W_{p}(G)
$$

follows readily. Suppose that equality is attained. Then $G$ has only one 2 -independent set and no $k$-independent set, where $k \geq 3$. Also, $D(G)=2$. Then, we have $G \cong G_{n}$. Conversely, if $G \cong G_{n}$, then the equality is attained.

For the case $D(G) \geq 3$ : Suppose that $u$ and $v$ are a pair of vertices in $G$ such that $d_{G}(u, v)=3$. Let $u x y v$ be a path of length 3 connecting $u$ and $v$ in $G$. Then $\{u, y\}$, $\{u, v\}$ and $\{x, v\}$ are 2 -independent sets of $G$. Therefore, every pair of vertices at distance 3 corresponds to three 2 -independent sets in $G$. Moreover, for any two paths connecting distinct pair vertices at distance 3 , they correspond to two different 2 -independent sets and one same 2-independent set, otherwise they correspond to three different 2 -independent sets. From this it follows that

$$
i(G ; 2) \geq 2 W_{p}(G)+1
$$

Therefore, by the definition of Merrifield-Simmons index, $i(G) \geq i(G ; 0)+i(G ; 1)+$ $i(G ; 2) \geq 1+n+2 W_{p}(G)+1=2+n+W_{p}(G),(2)$ follows readily.

Now, we check the equality condition. If $i(G ; 2)=2 W_{p}(G)+1$, by analysis, then any two paths of distinct pair vertices at distance 3 correspond to two different 2 -independent sets and one same 2 -independent set. If $i(G ; 3)=0, D(G)=3$. So $G \cong B_{2, n-2}$.

Conversely, if $G \cong B_{2, n-2}$, then we clearly have $i(G ; 2)=2 W_{p}(G)+1$ and $i(G ; 3)=$ 0 . So, the equality is attained if and only if $G \cong B_{2, n-2}$.

Theorem 5.3. Let $G$ be a connected graph with connected complement $\bar{G}$, then

$$
i(G)+i(\bar{G}) \geq 2 n+2 D(G)+2 D(\bar{G})-4
$$

with equality if and only if $G \cong P_{4}$.

Proof. By Lemma 5.1 and Lemma 5.2, the result is obvious.

Conjecture 5.4. Let $G$ be a connected graph with connected complement $\bar{G}$, then

$$
i(G)+i(\bar{G}) \leq 2+2 n+2^{n-3}+2^{n-2}
$$

with equality if and only if $G \cong S_{2, n-2}$. 
For a connected graph $G$ with connected complement $\bar{G}$, it is difficult to get the value of $\max \{i(G)+i(\bar{G})\}$. For $n \leq 5$, by enumeration and calculation, we can find $\max \{i(G)+$ $i(\bar{G})\}=\max \{i(T)+i(\bar{T})\}=i\left(S_{2, n-2}\right)+i\left(\overline{S_{2, n-2}}\right)$. If we do not consider the connectivity of the graph, we can get:

Theorem 5.5. Let $G$ be a simple graph of order $n$. If we do not consider the connectivity of the graph, then

$$
i(G)+i(\bar{G}) \leq 1+n+2^{n}
$$

with equality if and only if $G \cong K_{n}$ or $\bar{G} \cong K_{n}$.

Proof. Let $k, m \in \mathbb{N}$. Without loss of generality, we assume that $\alpha(G)>\alpha(\bar{G})$.

Every pair of vertices are not a 2 -independent set of $\bar{G}$, which compose of a 2 -independent set of $G$. Moreover, for any two vertices which do not compose of a 2 -independent set of $\bar{G}$, they compose of a 2 -independent set of $G$. Then, we have $i(G ; 2)+i(\bar{G} ; 2)=C_{n}^{2}$. Suppose $i(G ; 3)=k$. Since every three vertices which are a 3 -independent set of $G$ are not a 3 -independent set of $\bar{G}$, we have $i(\bar{G} ; 3) \leq C_{n}^{3}-k$. Therefore, we have

$$
i(G)+i(\bar{G}) \leq 2+2 n+C_{n}^{2}+C_{n}^{3}+\ldots+C_{n}^{\alpha(G)}=1+n+2^{n}-\sum_{i=\alpha(G)+1}^{n} C_{n}^{i}
$$

Now, we check the equality condition in (1). If $i(\bar{G} ; m)=C_{n}^{m}-i(G ; m)$, then for any $m$ vertices which are not an independent set of $\bar{G}$, they are an $m$-independent set of $G$. Then, $G$ is the empty graph, and $\bar{G} \cong K_{n}$. By the definition of $G$ and $\bar{G}$, we have

$$
i(G)+i(\bar{G}) \leq 1+n+2^{n}
$$

Obviously, for connected graph $G$ with a connected complement, $1+n+2^{n}$ is an upper bound on the maximum value of $i(G)+i(\bar{G})$. And, the lower bound on the maximum value of $i(G)+i(\bar{G})$ is $i\left(S_{2, n-2}\right)+i\left(\overline{S_{2, n-2}}\right)$. The difference between the upper bound and the lower bound is $5 \cdot 2^{n-3}-n-1$.

\section{Conclusions}

In this paper, we firstly establish a transformation on a simple graph that increases $i(G)$ and $i(\bar{G})$. Secondly, we prove the path $P_{n}$ and the double star $S_{2, n-2}$ are the extremal graphs which respectively reach the minimum and maximum value of $i(T)+i(\bar{T})$. Then, for unicyclic graphs $G$, we get that the cycle $C_{n}$ and the graph $O_{n-4,1,0}$ are the extremal graphs which respectively reach the minimum and maximum value of $i(G)+i(\bar{G})$. Finally, for connected graphs $G$, we find $i(G) \geq 2+n+2 W_{p}(G)$ with equality if and only if $G \cong G_{n}$ or $G \cong B_{2, n-2}$. Then we obtain $i(G)+i(\bar{G}) \geq 2 n+2 D(G)+2 D(\bar{G})-4$ with equality if and only if $G \cong P_{4}$. Also, we conjecture that the extremal graph which reaches the maximum value of $i(G)+i(\bar{G})$ is $S_{2, n-2}$. Which graph gives the maximum value on $i(G)+i(\bar{G})$ remains an open problem.

\section{References}

[1] M. Aouchiche and P. Hansen, A survey of Nordhaus-Gaddum type relations, Discrete Appl. Math. 161 (2013), 466-546, doi:10.1016/j.dam.2011.12.018. 
[2] H. Chen, R. Wu, G. Huang and H. Deng, Independent sets on the Towers of Hanoi graphs, Ars Math. Contemp. 12 (2017), 247-260, https : / / amc-journal.eu/index.php/amc/ article/view/783.

[3] S. Debroni, E. Delisle, W. Myrvold, A. Sethi, J. Whitney, J. Woodcock, P. W. Fowler, B. de La Vaissière and M. Deza, Maximum independent sets of the 120-cell and other regular polytopes, Ars Math. Contemp. 6 (2013), 197-210, https : / / amc-journal. eu/index. $\mathrm{php/amc/article/view/170.}$

[4] H. Deng and S. Chen, The extremal unicyclic graphs with respect to Hosoya index and Merrifield-Simmons index, MATCH Commun. Math. Comput. Chem. 59 (2008), 171190, http://match.pmf.kg.ac.rs/electronic_versions/Match59/n1/ match59n1_171-190.pdf.

[5] W. Du, X. Li and Y. Shi, Algorithms and extremal problem on Wiener polarity index, MATCH Commun. Math. Comput. Chem. 62 (2009), 235-244, http://match.pmf.kg.ac.rs/ electronic_versions/Match62/n1/match62n1_235-244.pdf.

[6] I. Gutman and O. E. Polansky, Mathematical Concepts in Organic Chemistry, Springer-Verlag, Berlin, 1986, doi:10.1007/978-3-642-70982-1.

[7] H. Hua and K. C. Das, On the Wiener polarity index of graphs, Appl. Math. Comput. 280 (2016), 162-167, doi:10.1016/j.amc.2016.01.043.

[8] H. Lei, T. Li, Y. Shi and H. Wang, Wiener polarity index and its generalization in trees, MATCH Commun. Math. Comput. Chem. 78 (2017), 199-212, http: / /match.pmf.kg.ac.rs/ electronic_versions/Match78/n1/match78n1_199-212.pdf.

[9] H.-H. Li, Q.-Q. Wu and I. Gutman, On ordering of complements of graphs with respect to matching numbers, Appl. Math. Comput. 282 (2016), 167-174, doi:10.1016/j.amc.2016.02. 004 .

[10] X. Li and Y. Mao, Nordhaus-Gaddum-type results for the generalized edge-connectivity of graphs, Discrete Appl. Math. 185 (2015), 102-112, doi:10.1016/j.dam.2014.12.009.

[11] X. Li, H. Zhao and I. Gutman, On the Merrifield-Simmons index of trees, MATCH Commun. Math. Comput. Chem. 54 (2005), 389-402, http://match.pmf.kg.ac.rs/ electronic_versions/Match54/n2/match54n2_389-402.pdf.

[12] R. E. Merrifield and H. E. Simmons, Topological Methods in Chemistry, A Wiley-Interscience Publication, Wiley, New York, 1989.

[13] E. A. Nordhaus and J. W. Gaddum, On complementary graphs, Amer. Math. Monthly 63 (1956), 175-177, doi:10.2307/2306658.

[14] A. S. Pedersen and P. D. Vestergaard, The number of independent sets in unicyclic graphs, Discrete Appl. Math. 152 (2005), 246-256, doi:10.1016/j.dam.2005.04.002.

[15] H. Prodinger and R. F. Tichy, Fibonacci numbers of graphs, Fibonacci Quart. 20 (1982), 16-21, https://www.fq. math.ca/Scanned/20-1/prodinger.pdf.

[16] D. Roth, On the hardness of approximate reasoning, Artif. Intell. 82 (1996), 273-302, doi: 10.1016/0004-3702(94)00092-1.

[17] S. Wagner and I. Gutman, Maxima and minima of the Hosoya index and the MerrifieldSimmons index: a survey of results and techniques, Acta Appl. Math. 112 (2010), 323-346, doi:10.1007/s10440-010-9575-5.

[18] D. B. West, Introduction to Graph Theory, Prentice Hall, Upper Saddle River, New Jersey, 2nd edition, 2001.

[19] Y. Zhang and Y. Hu, The Nordhaus-Gaddum-type inequality for the Wiener polarity index, Appl. Math. Comput. 273 (2016), 880-884, doi:10.1016/j.amc.2015.10.045. 
[20] Z. Zhu, The extremal unicyclic graphs with perfect matching with respect to Hosoya index and Merrifield-Simmons index, Ars Combin. 124 (2016), 277-287, http://www . combinatorialmath.ca/arscombinatoria/vol124.html.

[21] Z. Zhu, S. Li and L. Tan, Tricyclic graphs with maximum Merrifield-Simmons index, Discrete Appl. Math. 158 (2010), 204-212, doi:10.1016/j.dam.2009.09.001. 